\title{
PERSPECTIVAS INTERSECCIONALES Y COMPARADAS DE LA (IN) JUSTICIA DE GÉNERO Y VIOLENCIA EXTREMA CONTRA MUJERES AFRO E INDÍGENAS
}

INTERSECTIONAL AND COMPARATIVE PERSPECTIVES OF GENDER (IN) JUSTICE AND EXTREME VIOLENCE AGAINST AFRO AND INDIGENOUS WOMEN

\section{PERSPECTIVAS INTERSECCIONAIS E COMPARATIVAS DE (IN) JUSTIÇA DE GÊNERO E VIOLÊNCIA EXTREMA CONTRA MULHERES AFRO E INDÍGENAS}

Dolores Figueroa Romero Doctora en Sociología Catedrática CONACYT adscrita al Centro de Investigaciones y Estudios Superiores en Antropología Social dolores.figueroa@ciesas.edu.mx

México

Natalia De Marinis Doctora en Antropología Profesora- Investigadora Centro de Investigaciones y Estudios Superiores en Antropología Social - Golfo nataliademarinis@ ciesas.edu.mx México

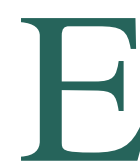

1 presente número especial contiene un grupo de ensayos que a partir de distintos contextos analizan y documentan las violencias extremas y estructurales que afectan la vida de mujeres indígenas $\mathrm{y}$ afrodescendientes, desde la costa atlántica de Nicaragua hasta las provincias centrales de
Canadá. Varios de los ensayos tratan de manera central el feminicidio y su relación con otras violencias a fin de aportar al entendimiento del feminicidio y las acciones en búsqueda de justicia y desde una lectura interseccional (WRIGHT, 2006; CASTAÑEDA, 2016; FIGUEROA, 2019). Los posicionamientos políticos y analíticos 
que inspiran estas reflexiones apuntan a entablar un diálogo crítico tanto con la academia como con el activismo feminista anti-feminicida desde una perspectiva enraizada que prioriza la observación empírica y el conocimiento denso de los contextos. Creemos importante facilitar diálogos que fertilicen y abran caminos de entendimiento que revelen y develen cegueras, prejuicios y miradas universalistas sobre experiencias de discriminación diferenciada, afectaciones inmateriales $\mathrm{y}$ reclamos de derechos desde racionalidades de sujetos políticos colectivos.

Partimos de la idea seminal de que es imperioso expandir el concepto de violencia de género y hablar de las violencias en plural, buscando hacer eco del entendimiento multifactorial de la violencia desde la experiencia de las mujeres indígenas y afrodescendientes (FIMI/IWIF, 2006; ECMIA, 2013). Pretendemos con lo anterior también aportar a los debates teóricos-metodológicos del enfoque interseccional tan en boga en las últimas décadas. La importancia de este giro en el abordaje de las opresiones/violencias reside en la necesidad de tomar distancia crítica del análisis feminista que prioriza la injusticia de género aislada de otras inequidades evitando así un mapeo más amplio e inclusivo de factores, de procesos, condiciones de vida e identidades sociales
(VIVEROS VIGOYA， 2016; SEODU HERR, 2020). La violencia feminicida debe verse de manera relacional y concatenada con otras violencias y procesos que enfatizan la vulnerabilidad y la discriminación contra mujeres de maneras desproporcionadas e injustas. Por ejemplo, al despuntar la guerra contra las drogas (2007-2017) y la militarización de ciertos territorios en el país, los feminicidios - antes circunscritos a ciudades fronterizas empezaron aparecer relacionados con la delincuencia común, el crimen organizado y la militarización (DATA CÍVICA, 2019; OVICOM, 2015). Ante la desarticulación de tejidos sociales básicos, el feminicidio y sobre todo el de mujeres racializadasdebe inscribirse en un mapa ampliado de relaciones que concatenan e interconectan en varias temporalidades y escalas de afectación (MARCHESE, 2019; DOCUMENTA DESDE ABAJO, 2020).

Violencias en plural - desde el punto de afectación colectiva de las mujeres indígenas- incluye las violencias resultado de discriminaciones estructurales como el despojo territorial, desplazamiento forzado, contaminación ambiental, pobreza, falta de acceso a salud o cualquier tipo de carencia material o inmaterial que de manera desproporcionada e injusta afecta a sectores social y económicamente marginados (ECMIA, 2013). Violencias en plural 
incluye las violencias cotidianas e invisibles que tienen un impacto acumulativo en la salud física y mental de indígenas, campesinos y trabajadores, tejiendo vulnerabilidades que conllevan a la muerte social (SCHEPER-HUGHES Y BOURGOIS, 2010). Las violencias en plural son inclusivas tanto de la violencia de género perpetuada contra las mujeres afrodescendientes e indígenas en la esfera doméstica, como las violencias que dañan el buen vivir de colectivos y grupos sociales diferenciados étnica y racialmente. Las mujeres indígenas de manera enfática han articulado y mostrado la necesidad de pensar la violencia en varias escalas de afectación donde lo individual-corporal es una parte de un todo compenetrado y conectado con otras relaciones y procesos (JACOBS y WILLIAMS, 2008). Todos estos elementos mencionados ilustran la naturaleza inclusiva -y podría decirse interseccional- de la conceptualización indígena, la cual se alimenta del trabajo político-activista indígena consistente que expande y confronta los referentes bajo los cuales las teorías feministas definen la violencia de género y diseñan políticas públicas para su prevención, sanción y eliminación (MERRY, 2006; GOEZT, 2007; SEODU HERR, 2020).

La confrontación al pensamiento feminista anti-violencia de género desde los colectivos de mujeres indígenas y afro tiene cinco elementos salientes, (1) cuestionar la universalidad/unicidad social de sujeto mujer (un tipo social no evidenciado); (2) La priorización de la lucha contra la violencia de género desde la mirada puesta únicamente en las relaciones interpersonales de poder entre hombres y mujeres; (3) la desarticulación de violencias colectivas de las experiencias de daño contra las mujeres indígenas como si su afectación estuviera desligada de los colectivos de pertenencia; (4) la descalificación de la cultura y orden social indígena y afrodescendiente como intrínsecamente violatorio de los derechos humanos de las mujeres; y (5) la colonialidad y jerarquías raciales, de clase y poder que caracterizan las prácticas de intervención en donde las mujeres diversas se convierten en sujetos a ser "salvados" (SIERRA, 2004; SEODU HERR, 2019; MERRY, 2006). En estos dos últimos puntos, añadiríamos un aspecto particular de la naturalización de la violencia en comunidades afrodescendientes en México que opera en dos sentidos, invisibiliza a la población de descendencia afro en las políticas nacionales y en el imaginario de la nación, a tiempo que escencializa el comportamiento de hombres y mujeres negros como intrínsecamente violentos y/o hiper-sexualizadas (MORENO FIGUEROA, 2020; ALCOCER, 2017). Estos elementos salientes de la crítica indígena a las ideas preconcebidas $\mathrm{y}$ 
occidental-céntricas del pensamiento feminista sobre las culturas "otras" también aterrizan en el campo de la lucha contra la violencia feminicida. Las preconcepciones racistas y sexistas - generan cegueras analíticas que impiden promover formas de erradicación de la violencia de género y feminicida que varían de acuerdo con los contextos, ya sean afro o indígenas. Más aún, las respuestas basadas en nociones individualistas liberales de derechos humanos dejan de lado las perspectivas locales de cuidado, resistencia y defensa desde las propias actoras y desde las opciones accesibles a nivel local.

Nuestro contrapunteo rescata el compromiso por aportar a los debates desde los feminismos de la diversidad (populares, comunitarios, indígenas y negros) a la redefinición del sujeto político del feminismo y sus varios sitios activistas. Pensando en nuestros ejes de colaboración creemos que podemos dar cuenta de la agencia política de mujeres indígenas y negras que en su labor de avanzar sus agendas construyen alianzas y alineamientos políticos con otros movimientos sociales en coaliciones de amplio espectro. Estas coaliciones son plataformas fundamentales para luchar contra el poder capitalista sistémico y cuestionar las hegemonía nacionales y globales desde las resistencias locales (VIVEROS VIGOYA, 2016).
En las últimas décadas el trabajo conceptual y activista de feministas trasnacionales y nacionales se ha concentrado en legitimar y universalizar los derechos humanos de las mujeres (COLE y PHILLIPS, 2008). En este marco de derechos, la lucha por una vida libre de violencia para las mujeres ha tomado un rol central en la homogenización a escala global de mecanismos y prescripciones contra la violencia de género que pretenden eliminar factores sistémicos y órdenes culturales que atentan contra la integridad física y moral de las mujeres (MERRY, 2006). Arropadas en el marco internacional de derechos humanos, el feminismo transnacional ha internacionalizado principios occidentales de justicia de género que prescriben la modificación de ideologías patriarcales de las culturas no occidentales a fin de eliminar las jerarquías sexo/genéricas que justifican la violencia contra mujeres racializadas (MORA, 2017). Este activismo -que sin duda ha expandido los derechos de las mujeres como ciudadanas en el mundo- tiende a ser particularmente adverso y negativo hacia las formas tradicionales de justicia indígena y a los espacios comunitarios de poder y reproducción social (SIERRA, 2004). Es por ello importante acentuar que el sitio de resistencia de las mujeres indígenas contra la violencia de género reside al centro de los colectivos a los cuales ellas pertenecen, 
aduciendo que la negociación de una vida libre de violencia pasa por el diálogo en concierto con su comunidad, y donde su red cercana de apoyo las acuerpa de manera sensible y cercana (SIEDER y BARRERA, 2017). Sin embargo, se vuelve preciso problematizar la escencialización de los valores y cohesión comunitarias en poblaciones que no han experimentado procesos organizativos cuestionadores y transformadores de prácticas comunitarias que vulneran los derechos de las mujeres. Muchas de las experiencias organizativas en movimientos amplios de reivindicación de derechos colectivos han demostrado que buena parte de los mal llamado "usos y costumbres" en regiones indígenas no son más que el resultado de los continuum de violencias coloniales que caracterizaron a los procesos de formación de Estado y asimilación indígena (CUMES, 2009).

En el contexto mexicano, el activismo feminista ha realizado una gran lucha para lograr una expansión de la ciudadanía política, social y económica de las mujeres (LAGARDE, 2008; ESPINOSA, 2009). En referencia a la violencia de género, las feministas mexicanas han desarrollado un trabajo muy importante en las ultimas décadas para visibilizar la problemática de feminicidio y el alarmante despunte de este tipo de homicidios en contextos de rápida urbanización y migración laboral fronteriza (WRIGHT, 2006; CASTAÑEDA, 2016). El feminicidio es la expresión más cruenta de violencia contra una mujer por ser mujer ya que culmina con la vida- y su definición conceptual y utilidad legal-punitiva ha sido producto de un trabajo muy arduo de feministas preocupadas por desnaturalizar la violencia patriarcal (FREGOSO Y BEJARANO, 2010). La lucha por visibilizar el feminicidio implicó la elaboración de diagnósticos exploratorios, la documentación estadística de los casos, el mapeo geográfico de su prevalencia y el diseño de políticas públicas para su prevención (LAGARDE, 2008; FIGUEROA, 2019). Tal pareciera que todos esos esfuerzos no fueron suficientes. Hoy en día el feminicidio se ha convertido en una pandemia, un tipo de violencia que debido a su alta prevalencia requiere redoblar esfuerzos para entender su especificidad y prevención desde el aporte de distintas disciplinas y enfoques que combinen lo cuantitativo con lo cualitativo (CASTAÑEDA, 2016; FIGUEROA, 2019).

Un argumento central de este número especial es que las perspectivas feministas sobre el feminicidio requieren ser revisitadas en función de cuatro elementos principales de crítica: (1) de-construir la lectura individualista de la experiencia de la violencia extrema por razones de género 
que no toma en cuenta la concatenación de "otras" violencias, estructurales y coloniales de largo aliento que aquejan a mujeres indígenas y afro-descendientes y a sus pueblos (CRENSHAW, 1991; GARCÍA DEL MORAL, 2017; RAZACK, 1998); (2) cuestionar la práctica de victimización de las mujeres indígenas sin respetar sus voces $\mathrm{y}$ sus propios referentes para responder y politizar la violencia (CABNAL, 2010; SEODU HERR, 2019). Este posicionamiento debe de ir equiparado con el ejercicio de de-construir la percepción de la mujer afrodescendiente como sexualmente liberada, activa y promiscua; (3) la documentación de las violencias debe hacerse como resultado de la aplicación de la exploración de metodologías de investigación interseccional, empírica y políticamente situada que apuesten por el entendimiento de otras formas alternativas y comunitarias de justicia de género y resarcimiento del daño; y (4) enriquecer la tendencia a correlacionar el feminicidio con otro tipo de violencias extremas como la desaparición forzada, la trata, el secuestro, la extorsión, el trafico de estupefacientes y el desplazamiento forzado, ya que se deja de lado los impactos de la pobreza estructural y el racismo social contra los pueblos indígenas y afrodescendientes. En este aspecto, la dimensión territorial e histórica adquiere particular relevancia para comprender las características que adquiere violencia institucional, los nexos entre actores estatales y el crimen organizado, y la enorme impunidad en las que se traducen estas complicidades (OVICOM, 2015; MORA, 2013).

Sin ánimo de descalificar este trabajo arduo de conceptualización y activismo legal feminista, lo cierto es que hereda un fuerte prejuicio colonial mestizo que señala a las comunidades indígenas, sus culturas y sus autoridades como intrínsecamente violatorias a los derechos de las mujeres naturalizando la violencia en sus territorios y subestimando el llamado a entender y documentar de maneras densas y contextuales (HERNÁNDEZ, 2004; SIERRA, 2004). En ese ejercicio externo e intervencionista de señalar a la cultura indígena -y en su particular sentido también la afrodescendiente- como violatoria a los derechos humanos de las mujeres, se deja de lado el entendimiento de los efectos sociales de la pobreza estructural, el colonialismo de larga data y el despojo neoliberal que opera en territorios indígenas y genera tensiones extra e intra-comunitarias que invariablemente devienen en relaciones de poder abusivas en casa. Es por ello que, en el concierto actual de la lucha antifeminicida, asistimos a un importante subregistro sobre las violencias extremas contra mujeres en territorios atravesados por una acumulación histórica de violencias 
y despojos, situación agravada por las embestidas más recientes del crimen organizado y la militarización (DE MARINIS y FIGUEROA, 2019; HERNÁNDEZ, 2017). El silencio alrededor de los feminicidios y asesinatos de mujeres racializadas es una expresión de la normalización de la violencia de género en estas regiones y, de manera general, sobre estos cuerpos; del miedo y las amenazas que generan la criminalización y respuestas militarizadas en estas regiones; de los cuestionables procesos de justicia que se encaran ante los asesinatos de mujeres racializadas en regiones con altos índices de corrupción e impunidad; pero también, del ocultamiento de las identidades de las víctimas en las estadísticas oficiales y los vacíos de algunos intentos de documentación no oficiales (LUCCHESI y ECHO-HAWK, 2018; DEL JURADO y DON JUAN, 2019). Este silencio, sin embargo, está siendo enfrentado desde las organizaciones de mujeres que acompañan a mujeres víctimas de violencia y que buscan denunciar la manera en que sus cuerpos se vuelven territorios de construcción soberana, con marcas y mensajes que ponen de manifiesto -junto con el género- otras condiciones de opresión (SEGATO, 2013; VIVEROS

https://lasillarota.com/lacaderadeeva/feminicidios-deindigenas-las-muertes-silenciadas-violencia-generofeminicidios-indigenas/312887
VIGOYA, 2016). Sí hay feminicidios de mujeres indígenas y afrodescendientes, pero en la mayoría de los casos no sabemos cuántas son, quiénes son, en qué circunstancia fueron atacadas y los móviles. ${ }^{1}$ Varios de los ensayos incluidos en este número responden a estas preguntas, y reconocemos que se requieren más trabajos de investigación en los territorios que recojan la voz de las y los defensores y familiares de las víctimas de feminicidio.

\section{Elementos conceptuales $\mathbf{y}$ metodológicos salientes y con los cuales dialogamos y buscamos aportar}

En función de nuestros estudios de caso y de las múltiples trayectorias de investigación colaborativa, retomamos de manera nodal el concepto de interseccionalidad por su potencialidad, ductibilidad y fertilidad para dar sentido a la conjunción de violencias y órdenes discriminatorios, la construcción de identidades sociales diferenciadas y el trabajo activista por la justicia social y de género (CRENSHAW, 1991; HANCOCK, 2007; VIVEROS VIGOYA, 2016). La interseccionalidad es reconocida ahora como 
un importante aporte teórico-conceptual a la teoría feminista, y ha desarrollado diversas apuestas de exploración metodológica que han sido adoptadas de maneras diferentes, acorde a distintos tipos de feminismos y geografias de lucha. De gran importancia ha sido desarrollar y posicionar este paradigma que ayuda conectar dos polos de análisis: por un lado, la conjunción de opresiones estructurales, en relación y en conexión a la experiencia microsocial de discriminación de colectivos con identidades sociales diferenciadas (VIVEROS VIGOYA, 2016). En nuestro caso encontramos importante retomar la riqueza de la academia feminista americana negra y su preocupación por el análisis de las políticas públicas y la intersección de opresiones estructurales como son el género y la raza, ello aunado con lo que develan los estudios indígenas decoloniales, $\mathrm{y}$ el feminismo comunitario latinoamericano que muestra la experiencia de la violencia colonial y el patriarcado capitalista desde la interpelación femenina popular en el sur global (CUMES, 2014; TZUL TZUL, 2016; CABNAL, 2010).

No hay el suficiente espacio para explayarnos en mostrar la genealogía de los múltiples aportes teóricos y metodológicos del concepto de interseccionalidad, más bien lo que queremos resaltar son los ejes de análisis más fructíferos que consideramos tocan $\mathrm{y}$ hacen eco de los casos trabajados en los ensayos del dossier: Primero, un eje trata de cómo las identidades sociales de grupos sociales marginalizados se constituyen y construyen de manera fluida y relacional, incorporando distintos factores marcadores como es la etnicidad, la raza, el género, la clase y el grupo. Segundo y fundamentalmente ligado al primer eje, está la interseccionalidad de las opresiones y determinaciones estructurales e históricas de los contextos específicos. Tercero, la interaccionalidad cruza distintos niveles y escalas de análisis (y ello implica tanto escalas geográficas $\mathrm{y}$ cuerpo/espacio como campos de análisis que se tocan). Cuarto, la interseccionalidad política del trabajo de activistas que como resultado de sus estrategias de incidencia invisibilizan los reclamos de mujeres de identidades subalternas como indígenas $\mathrm{y}$ afrodescendientes (CRENSHAW, 1991). Y quinto, una perspectiva interseccional que ayude a empujar diálogos constructivos y reveladores de las cegueras y puntos ciegos de las acciones políticas y léxicos reivindicatorios (WRIGHT, 2017; LYKKE, 2011).

Específicamente en este número se explorarán las potencialidades analíticas del concepto de interseccionalidad política acuñado por Crenshaw (1991) y Lykke (2011) para entender la complejidad de la posicionalidad de colectivos que politizan su identidad y experiencia de violencia en los márgenes de los activismos hegemónicos -ya sea feministas, étnicos, o de derechos 
humanos. Esta perspectiva refiere dar sentido al desencuentro de las agendas activistas -en nuestros casos el activismo anti-violencia feminicida y de género con el activismo indianista- que por lo general toman caminos paralelos o se encuentran de manera contradictoria y que generan silencios sobre violencias y aspectos que son puestos en un segundo plano o no son incorporadas en su quehacer activista. Digamos por ejemplo, para el activismo anti-feminicida es importante resaltar la relación individualizada víctima-victimario de la experiencia de violencia sexo/genérica; y por el contrario, para las activistas indígenas su acción de denuncia se expande hacia las violencias colectivas que ocurren en territorios $y$ espacios geográficos concretos.

Un tema recurrente en varios de los ensayos de nuestro compendio es explorar qué aporta la perspectiva interseccional a la lectura crítica de las políticas públicas para prevenir, sancionar y erradicar los feminicidios. Como ya mencionamos, la violencia de género es una problemática de análisis recurrente y políticamente definitoria del activismo feminista en México al igual que en otros países de las Américas (MOLYNEAUX, 2007; JARAMILLO, 2020). En el campo del acceso a la justicia y las definiciones de las injusticias contra mujeres, la creación de leyes que castiguen a los actores que discriminan basado en la diferencia de género ha sido un eje central (NUÑEZ, 2018). Exigir y castigar las injusticas y crímenes de género desde la mirada legal y punitiva ha sido central para el feminismo institucional en función de crear prescripciones, protocolos y mecanismos en las instituciones del gobierno. Sin embargo, los márgenes de aplicabilidad y eficiencia en resolución son enormes. Impunidad es lo que en la mayoría de los casos ocurre con las víctimas de violencia de género, de todas las edades, de todas las etnicidades, de todos los estratos sociales $y$ de todos confines geográficos (LAGARDE, 2008; DEL MORAL, 2017; DE MARINIS, 2019). Al poner en cuestionamiento los límites de la justicia institucional queremos mostrar que hay muchas experiencias de discriminación, afectación y vulneración de derechos que no pasan por la justicia del Estado, y que las construcciones de daño y sentidos de justicia tome lugar en varios espacios sociales, entre ellos el orden social de los pueblos y la perspectiva de justicia comunitaria (FIGUEROA Y SIERRA, 2019).

En términos metodológicos diríamos que la apuesta de este número es contribuir al debate sobre la generación de información desde los contextos y desde los actores demandantes de justicia. Nos sumamos a la tarea de enriquecer el paradigma de la interseccionalidad desde estudios empíricos y etnográficos, pues congeniamos con la idea 
de que las estrategias metodológicas interseccionales son tan necesaria como el propio ejercicio de interpretación teórica. En específico, queremos fortalecer y robustecer desde la disciplina antropológica la mirada cualitativa que rescate la interseccionalidad de la realidad y la interseccionalidad en la construcción del dato en la investigación.

De Hancock (2007) reconocemos que la metodología interseccional implica generar una estrategia de recolección de datos de maneras alternativas (p. 66). Lo cual implica desechar investigación que se ha realizado con métodos tradicionales y unitarios. Tomar en cuenta la intersección de diferentes campos de poder como hegemonía y cultura, derecho e instituciones sociales, Estado y prácticas administrativas y disciplinarias contra población minoritaria; interacciones rutinarias en instituciones de gobierno $\mathrm{y}$ racismo, etc. Desde nuestros abordajes cualitativos debemos de experimentar con nuevos medios para rescatar la experiencia interseccional de los actores sociales, los contextos y las políticas de la identidad. Pensar detenidamente cómo colectar información y diseñar investigación interseccional tomando en consideración muchas más variables desde una perspectiva relacional. Implica también investigaciones rigurosas que respondan a las necesidades de colaboración y diálogo con los y las actores a fin de responder al reto cognitivo de justicia.
Un elemento importante de la colaboración es pensar la interseccionalidad metodológica desde la necesidad de las activistas afro e indígenas organizadas. El cuestionamiento y la preocupación por el subregistro de las violencias extremas y el análisis de realidades que no son visibles, como las violencias cotidianas, requieren resolver retos metodológicos que nos lleven a desentrañar el origen de las cegueras y la búsqueda de maneras para subsanarla, tanto en lo cualitativo como en lo conceptual. Hancock (2007) nos dice que una tarea vital es desmontar preconcepciones que señalan la patología de individuos desligada del condicionamiento institucional. Con patologías ella refiere a la construcción racista de la violencia en la comunidad afrodescendiente americana, pero para otros contextos latinoamericanos significa desmontar la naturalización de comportamientos violentos en culturas $\mathrm{y} / \mathrm{o}$ razas, la falta de agencia/consciencia feminista en mujeres indígenas, la hipersexualidad e independencia en mujeres afro, etc. Todos los prejuicios de clase y coloniales que marcan la forma de operar y racionalizar del feminismo urbano y hegemónico que filtra y vela la información sobre grupos sociales que son racializados, y en una línea de modernización de comportamientos respetuosos de los derechos humanos de las mujeres quedan estigmatizados como retrasados y violentos. 
La preocupación por el subregistro de los feminicidios de mujeres indígenas $\mathrm{y}$ afrodescendientes es una pieza clave para interpelar al feminismo oficial y las formas en que se produce información cuantitativa $\mathrm{y}$ cualitativa sobre el feminicidio en México y en otros países de las Américas (GARCÍADEL MORAL, 2017; NEWMAN y GARCÍA-DEL MORAL, 2018). Esto es un reto político y metodológico para varias articulistas de este número, y que tratan de entender las violencias contra las mujeres racializadas desde las violencias más extremas hasta las opresiones menos reconocidas -pobreza estructural y despojo patrimonial- como tradicionalmente ligadas o interconectadas con la violencia doméstica.

Entendemos la documentación desde un sentido amplio, pues no sólo nos referimos a la producción de datos estadísticos sobre casos y hechos violentos (desaparición forzada y feminicidio/homicidio doloso) sino producir información contextual, histórica, etnográfica y culturalmente apropiada sobre los escenarios, los actores y los hechos violentos que aquejan a mujeres indígenas y afros y sus pueblos. Esta documentación apunta a proveer una descripción densa histórica-contextual y etnográfica que políticamente revele, desnaturalice, dignifique $y$ que articule otras interpretaciones sobre las víctimas, victimarios, actores circundantes y agentes del estado. Una documentación que ayude a entender las violencias no como actos aislados y fortuitos sino como parte de un entramado social, político y económicoestructural más amplio donde hay una corresponsabilidad por parte del Estado en no proteger, no detener, no sanar, no restaurar, no atender, misma que varía de acuerdo con los territorios y las personas.

Partimos del principio que la elaboración de bases de datos sobre la violencia contra mujeres diversas es un reto metodológico y técnico. Por ello es que vemos necesario explorar con varias metodologías de levantamiento de datos, medios para la sistematización de la información y la combinación de varias formas de trabajo y los resultados de distintos equipos de investigacion. La documentación de las violencias contra mujeres indígenas y afros es un tarea que requiere la aplicación tanto de metodologías cuantitativas como cualitativas. Por una parte, la implementacion de métodos cuantitativos en la medición de la violencia de género debe ser sensible a la construcción de indicadores y datos estadísticos que reflejen la identidad étnica y racial de las víctimas. $\mathrm{Y}$ por otra, las metodologías cualitativas deben dar centralidad al entendimiento de los contextos y el aporte etnográfico del orden cotidiano y microsocial. 


\section{Las contribuciones}

La apuesta de este número es enriquecer desde perspectivas comparadas el uso y adaptación del enfoque interseccional feminista desde una perspectiva múltiple y en varias escalas: en primera instancia, queremos compartir los resultados de estudios empíricos y etnográficos realizados sobre las violencias extremas y cotidianas contra mujeres indígenas y afrodescendientes en México, Centroamérica y Canadá; nuestros trabajos están inspirados en un compromiso por experimentar y construir nuevas rutas metodológicas de investigación que permitan producir información densa, contextual y de primera mano, y con ello contribuir al debate metodológico de cómo hacer investigación interseccional $\mathrm{y}$ comprometida; de igual forma alimentamos distintos esfuerzos de teorización sobre la interseccionalidad de violencias desde las experiencias de mujeres racializadas y sus reclamos de derechos como un sitio privilegiado que permite develar y revelar verdades parciales, prejuicios institucionales y racismos entronizados. Las perspectivas comparadas nos permiten ubicar los usos y circulaciones de categorías para la construcción de demandas e interpelación con el Estado y en la definición misma de los agravios; así mismo, nos ayuda a comprender las posibilidades y constricciones de las categorías vuelta letra legal, sobre todo cuando operan en escenarios de elevada impunidad y complicidades patriarcales. Las metodologías propuestas en los artículos, si bien comparten los abordajes situados y colaborativos, representan apuestas diversas e involucramientos de muchos tipos, que van desde los trabajos de memorias y documentación hasta la elaboración de peritajes expertos en procesos judiciales.

En el artículo "Diálogos binacionales sobre los retos para documentar la(s) violencia(s) contra mujeres indígenas en México y Canadá”, sus autoras presentan una serie de reflexiones de orden metodológico y político surgidas de una mesa compartida en el Congreso de la Asociación de Estudios Latinoamericanos (LASA) en mayo de 2020. En el artículo se proyectan aportes a la reflexión comparativa, indígena e interseccional sobre las metodologías cuantitativas y cualitativas - para documentar las violencias extremas y feminicidios en México y Canadá. Norma Donjuan Pérez y Patricia Torres Sandoval, como integrantes de la Coordinadora Nacional de Mujeres Indígenas (CONAMI), y Dolores Figueroa Romero y Vivian Jiménez Estrada como académicas comprometidas en diferentes procesos en México y Canadá, desde la Indigenous Women's Anti-Violence Task Force (IWAVTF), comparten un análisis transnacional basado en un diálogo norte-sur 
sobre estrategias propias y culturalmente situadas, para visibilizar y comprender las experiencias de violencia colonial y patriarcal infligida contra mujeres Indígenas en los territorios de la "Isla Tortuga" o Norteamérica y en Abya Yala en general.

En el artículo "Feminicidios de mujeres indígenas en clave interseccional: análisis a partir de un trabajo de documentación colaborativa con mujeres nahuas organizadas en Zongolica”, Natalia De Marinis realiza un recorrido por la construcción, usos y destinos de la categoría feminicidio en México para comprender cómo ha sido apropiado por mujeres indígenas de la región nahua de Zongolica en Veracruz, y se pregunta qué tanto es útil para comprender y documentar los marcos territoriales y de violencias históricas acumuladas en los territorios. Desde un trabajo colaborativo de documentación con mujeres nahuas organizadas, la autora plantea una crítica a la manera en que la categoría, al ubicarse en un sentido más bien interpersonal de las relaciones desiguales sexo-genéricas, limita los sentidos múltiples e interseccionales de violencia que develan las memorias de las mujeres sobre estos hechos.

Las contribuciones del artículo de Paulina García-Del Moral titulado: "Mujeres y niñas indígenas desaparecidas y asesinadas: Femicidio, Feminicidio, y Genocidio en el contexto canadiense" se centran en las limitaciones de categorías conceptuales y legales en torno al asesinato de mujeres indígenas. Parte de un análisis del contexto canadiense, donde los asesinatos y desapariciones de mujeres y niñas indígenas fueron caracterizados recientemente como genocidio, y donde el término feminicidio no es una categoría reconocida. Discute en su artículo tres explicaciones por las cuales la categoría feminicidio, empujada por cierto sector de la academia feminista, no es apropiada por las organizaciones indígenas. En primer lugar, por la ausencia de una perspectiva interseccional en la formulación histórica de la categoría feminicidio; en segundo lugar, por la ausencia histórica de alianzas entre el feminismo en Canadá y las mujeres indígenas; por último, por las barreras de orden lingüísticas y de poder que restringen el flujo de conocimiento del sur al norte global.

En el artículo "Mujeres indígenas rompen el silencio y exigen justicia. Tribunal del Conciencia sobre Violencias de Género en México", María Teresa Sierra y Dolores Figueroa Romero analizan los testimonios de mujeres indígenas de la Montaña de Guerrero compartidos en diferentes Tribunales de Conciencia organizados por el Centro de Derechos Humanos de la Montaña Tlachinollan. Las autoras analizan, a partir de los testimonios de las mujeres ante un público amplio que incluyó a miembros de la 
sociedad civil, academia, abogados, entre otros, la manera en que los mismos visibilizaron la discriminación estructural, patriarcal e institucional. Con base en algunos de los casos que se presentaron en los distintos Tribunales de Conciencia y muy especialmente los que tuvieron alguna continuidad, las autoras proponen una lectura antropológica de la problemática de la violencia extrema que viven las mujeres indígenas en Guerrero, los efectos racializantes y excluyentes que enfrentan en la búsqueda de justicia y lo que revela del cúmulo de opresiones que afectan sus vidas y la de sus comunidades.

Marisol Alcocer Perulero analiza en su artículo “¿Feminicidio de afrodescendientes en México? Lo que no se nombra no existe" los vacíos en la documentación y registro de prensa de las violencias contra las mujeres afromexicanas en el estado de Guerrero. Parte del argumento de que la violencia que viven las mujeres afromexicanas es multifactorial, a lo que se le agrega un contexto con múltiples problemáticas sociales e históricas, como el crimen organizado, la militarización histórica, la presencia de grupos organizados para la autodefensa, entre otros, lo que da una impronta particular al análisis e interpretación de la violencia que experimentan mujeres racializadas. El gran vacío que describe la autora en relación con la información sobre las víctimas, su condición étnica, el lugar de origen, entre otras, es aún más palpable para el caso de mujeres afromexicanas, identidad diferenciada recién legalmente reconocida en el país. Marisol Alcocer propone que, si bien la aproximación estadística es importante para entender la problemática del feminicidio, es preciso tener un acercamiento cualitativo, para reflexionar en torno a los significados y las interpretaciones que tienen las mujeres afromexicanas respecto los agravios que viven.

$$
\text { Arelly Barbeyto, Nora Sánchez, Katy }
$$
Cedeño y Selmira Flores proponen un artículo acerca de los conflictos por invasión irregular en las tierras comunitarias indígenas. Titulado: "Las voces de las mujeres miskitu en la conflictividad del territorio Wangki Twi Tasba Raya en la Región Autónoma del Caribe Norte de Nicaragua", las autoras nos brindan una interpretación de los efectos de la presión de los frentes colonizadores de población campesina en la vida de las mujeres indígenas. Denunciando la injusticia por el despojo del asentamiento histórico contra la población Miskitu, el artículo abona a la discusión acerca de la manera en que la conflictividad territorial restringe derechos de las mujeres al acceso, posesión y uso de la tierra, y genera agravios multidimensionales contra las mujeres y la 
vida comunitaria. Las autoras lo analizan a partir de una serie de entrevistas a mujeres que narran las violencias y reflexionan sobre los procesos de construcción y resistencia colectiva que han encarado.

Por su parte, Perla Fragoso Lugo en su artículo: "Los pactos patriarcales en el ocultamiento de un delito: Feminicidio y violaciones a los derechos humanos en Chiapas" analiza las limitaciones conceptuales contenidas no tanto en la categoría de feminicidio en sí, sino en las tranformaciones que sufre desde su desplazamiento hacia la categoría legal e interpretación en los procesos judiciales. A través de una descripción detallada del proceso por el cual Francisca -una mujer indígena tsoltsil en Chiapas- fue acusada del asesinato de su sobrina, y de los elementos clave de un peritaje experto que la misma autora estructuró su para su defensa, Fragoso Lugo analiza cómo una mujer indígena se vuelve presa fácil de las complicidades, pactos patriarcales e impunidad que caracterizan las instituciones de justicia en regiones indígenas. Analiza el proceso por que el Florencia fue injustamente acusada del asesinato de su sobrina a partir de una mirada que intersecta los continuиm de violencias estructurales, comunitarias, institucionales e interpersonales y que permiten comprender la invisibilización y el subregistro de los feminicidios de mujeres indígenas.
Finalmente, el dossier incorpora el testimonio de Rubí Alondra, mujer nicaragüense de Masaya, luchadora social que enfrentó al régimen de Ortega y cuya participación activa en el levantamiento de 2018 le costó el exilio. Desde Canadá, país que cobijó su demanda de asilo político, nos narra cómo se vivieron esos momentos de levantamiento y rebeldía, cómo se vivieron el amor y la alegría de saberse juntos peleando por algo justo, y cuál fue la respuesta que se orquestó mediante ataques militares y paramilitares que acabaron con la vida de muchas personas, instalando el silencio, el terror y orillando al exilio de otras cuantas. ¿Cómo se intersectan las violencias en los cuerpos de las mujeres de Nicaragua? ¿Cómo se construyen las resistencias desde esas intersecciones? ¿De qué manera las rebeliones de un país bajo un régimen autoritario han permitido sueños y deseos que reviertan los órdenes de género y de violencia que sostienen? La potencia de su narrativa, rebosante de detalles de la violencia y la resistencia, nos lleva no sólo a un viaje por los intersticios de las acciones micro-políticas de una rebelión sin precedentes, sino que también nos muestra porqué la circulación de su testimonio para la construcción de la memoria se vuelve un arma poderosa de resistencia, de construcción colectiva y de imaginación de otros futuros. 


\section{Referências bibliográficas}

ALCOCER, Marisol. "Los motivos de Jano: ejercicio y contención de la violencia física, sexual y feminicidio contra mujeres en dos comunidades afromexicanas de la Costa Chica de Guerrero". Tesis Doctoral en Ciencias Sociales. FLACSO: México, 2017.

CABNAL, Lorena. "Acercamiento a la construcción del pensamiento epistémico de las mujeres indígenas feministas comunitarias de Abya Yala", En Feminismo diversos: el feminismo comunitario. ACSUR Las Segovias, 2010, pp. 10-26.

CASTAÑEDA, Marta Patricia. "Feminicide in Mexico: An approach through academic, activist and artistic work". Current Sociology, v. 64 n. 7, pp. 1054-1070, 2016.

COLE, Sally y PHILLIPS, Lynn. "The Violence against Women Campaigns in Latin America: New Feminist Alliances". Feminist Criminology, v. 3 n. 2, pp. 145-168, 2008.

CRENSHAW, Kimberle. "Mapping the Margins: Intersectionality, Identity Politics and Violence against Women of Color". Stanford Law Review, v. 43 n. 6, pp. 12411299, 1991.

CUMES, Aura. "Sufrimos vergüenza: Mujeres K'iché frente a la justicia comunitaria". Desacatos, v. 31, pp. 99-114, 2009.

CUMES, Aura. "Multiculturalismos, género y feminismos: mujeres diversas, luchas complejas." En ESPINOSA MIÑOSO, Yuderkys, GÓMEZ CORREAL, Diana y OCHOA MUÑOZ, Karina. Tejiendo de otro modo: Feminismo, epistemología y apuestas descoloniales en Abya Yala. Cauca:

Universidad del Cauca, pp. 237-352, 2014.

DATA CÍVICA. "Claves para entender y prevenir los asesinatos de mujeres en México", 2019. Disponible en https://datacivica.org/assets/pdf/claves-para- entender-y-prevenir-los-asesinatos-demujeres-en-mexico.pdf

DE MARINIS, Natalia. Desplazadas por la guerra. Estado, género y violencia en la región triqui. México: CIESAS, 2019.

DE MARINIS, Natalia y FIGUEROA Romero, Dolores. "Violencias contra mujeres indígenas y afrodescencientes: Reflexiones desde la investigación, la acción y la documentación". Ichan Tecolotl CIESAS, febrero, 2019. Disponible en https://ichan.ciesas.edu.mx/2019/02/

DEL JURADO, Fabiola y Norma DONJUAN PÉREZ. "Emergencia comunitaria de género. Respuesta de las mujeres indígenas a las múltiples violencias y el despojo del territorio". Ichan Tecolotl CIESAS, febrero, 2019. Disponible en https://ichan.ciesas.edu.mx/puntos-deencuentro-anteriores/emergenciacomunitaria-de-genero-respuesta-de-lasmujeres-indigenas-a-las-multiples-violenciasy-el-despojo-del-territorio/

DOCUMENTA DESDE ABAJO. AntiManual: Para la realizacion de talleres con sobrevivientes de graves violaciones a derechos humanos a partir de un enfoque territoral y participativo. Ciudad de México, 2020.

ECMIA, 2013. Violencias y mujeres indígenas. Disponible en http://ecmia.org/index.php/publicaciones/177violencias-y-mujeres-indigenas

ESPINOSA DAMIAN, Gisela. Cuatro vertientes del feminismo en México. Diversidad de rutas y cruce de caminos. México: UAM, 2009.

FIGUEROA ROMERO, Dolores y SIERRA CAMACHO, María Teresa. "Alertas de género y mujeres indígenas: interpelando las políticas públicas desde los contextos comunitarios en Guerrero". Canadian Journal of Latin American and Caribbean Studies, v. 45, n. 1, pp. 26-44, 2020. 
FIGUEROA ROMERO, Dolores. "Políticas de Feminicidio en México: Perspectivas interseccionales de mujeres indígenas para reconsiderar su definición teórica-legal y las metodología de recolección de datos", Journal of International Women Studies, v. $20 \mathrm{n}$.

8, pp. 64-86, 2019.

FREGOSO, Rosa-Linda y BEJARANO, Cynthia. Terrorizing Women: Feminicide in the Americas. Duke University Press, 2010.

GARCÍA-DEL MORAL, Paulina y NEUMANN, Pamela. "The Making and Unmaking of Feminicidio/Femicidio Laws in Mexico and Nicaragua". Law \& Society Review, v. 53 n. 2, pp. 452-486, 2018.

GARCÍA-DEL MORAL, Paulina. "The Murders of Indigenous Women in Canada as Feminicides: Toward a Decolonial Intersectional Reconceptualization of Femicide". Signs: Journal of Women in Culture and Society. vol. 43, no. 4, pp. 929954, 2018.

GOEZT, Anne. "Gender Justice, citizenship, rights. Fundamental concepts, debates and new research directions" En MAITRAYEE, Mukhopadhyay y SINGH, Navsharan. Gender justice, Citizenship and Development, IDRC: Ottawa, pp. 13-46, 2007.

HANCOCK, Ange-Marie. "When multiplication does not equal quick addition: Examining Intersectionality as a Research Paradigm". Perspectives on Politics, v. 5, n. 1, pp. 63-79, 2007.

HERNÁNDEZ CASTILLO, Aida. "La Diferencia en el Debate: Las Políticas de Identidades en Tiempos del PAN". En HERNÁNDEZ, Aida, PAZ, Sarela y SIERRA, María Teresa. El Estado y los indígenas en tiempos del PAN: Neoindigenismo, legalidad e identidad. CIESAS: México, pp.- 287-306, 2004.

HERNÁNDEZ CASTILLO, Aida. "La guerra contra el narco. Violencia de Género, militarización y criminalización de los pueblos indígenas". En BASTOS, Santiago; SIERRA, María Teresa. Estado y pueblos indígenas en México. La disputa por la justicia y los derechos. Colección México. México: CIESAS. Pp. 244-267, 2017.

IIWF/FIMI. Mairin Iwanka Raya. "Indigenous Women Stand Against Violence. A Companion Report to the United Nations Secretary". General Study on Violence Against Women. Managua: UNIFEM, 2006.

JACOBS, Beverley y WILLIAMS, Andrea J. "Legacy of Residential Schools: Missing and Murdered Aboriginal Women". En BRANT CASTELLANO, Marlene, ARCHIBALD, Linda y DEGAGNÉ, Mike. From Truth to Reconciliation: Transforming the Legacy of Residential Schools. Ottawa: Aboriginal Healing Foundation, pp. 119-142, 2008.

JARAMILLO SIERRA, Isabel. "Latin American Feminist Legal Theory. Taking Multiple Subordinations Seriously". En SIEDER, Rachel; ANSOLABEHERE, Karina y ALONSO, Tatiana. Routledge Handbook of Law and Society in Latin America. New York: Routledge, pp- 111-125, 2019.

LAGARDE, Marcela. "Antropología, Feminismo y Politica: Violencia Feminicida y Derechos Humanos de las Mujeres". En BULLEN, Margaret y DIEZ MINTEGUI, María del Carmen. Retos Teóricos y Nuevas Prácticas, Mexico: UNAM, pp. 209-239, 2008.

LUCCHESI, Annita y ECHO-HAWK, Abigail. Missing and Murdered Indigenous Women and Girls. Urban Indian Health Institute/Sovereing Bodies Institute: USA, 2018.

LYKKE, Nina. "Intersectional Analysis: Black Box or Useful Critical Feminist Thinking Technology?'. En LUTZ, Helma, HERRERA VIVAR, María Teresa y SUPIK, Linda. Framing Intersectionality Debates on 
a Multi-faceted Concept in Gender Studies. Surrey: Ashgate, pp. 207-220, 2011.

MARCHESE, Giulia. "Del cuerpo en el territorio al cuerpo- territorio: Elementos para una genealogía feminista latinoamericana de la crítica a la violencia". EntreDiversidades, v. 6 n. 2, pp. 9-41, 2019

MERRY, Sally Engle. Human rights and gender violence: translating international law into local justice. Chicago: University of Chicago Press, 2006.

MOLYNEAUX, Maxime. "Reconfiguring citizenship: Research Perspectives on Gender Justice in the Latin American and Caribbean Region", En MAITRAYEE, Mukhopadhyay y SINGH, Navsharan. Gender justice, Citizenship and Development. IDRC: Ottawa, opp. 47-90, 2007.

MORA, Mariana. "La criminalización de la pobreza y los efectos estatales de la seguridad neoliberal: Reflexiones desde la Montaña, Guerrero". Revista de Estudios e Pesquisas sobre as Américas, Universidade de BrasíliaUnB, v. 7 n. 2, pp. $174-208,2013$.

MORA, Mariana. "Voces desde los silencios. Mujeres indígenas, seguridad y derechos frente a las violencias en la Montaña de Guerrero". En SIEDER, Rachel. Exigiendo justicia y seguridad. Mujeres indígenas y pluralidades legales en América Latina. México: CIESAS, pp. 315-398, 2017.

MORENO FIGUEROA, Mónica. "El giro hacia el racismo y anti-racismo. El Mestizaje y racismo anti-negro en México". Ponencia en el panel "Genealogías, abordajes y debates del racismo", Congreso Latin American Association (LASA) 2020. Disponible en https://www.facebook.com/LatinAmericanSt udiesAssociation/videos/849317192243602. 31:16 minutos/ 1:49:55.

NÚÑEZ, Lucía. El género en la ley penal: crítica feminista de la ilusión punitiva. México:UNAM, 2018.
OVICOM. "Informe de Homicidios Dolores de Mujeres del Estado de Guerrero (20052015)". El Observatorio de Violencia Contra las Mujeres "Hannah Arendt": Guerrero, 2015.

RAZACK, Sherene. "Race, Space, and Prostitution: The Making of the Bourgeois Subject". Canadian Journal of Women and the Law, v. 10 n. 2, pp. 121-156, 1998.

RAZACK, Sherene. "Gendering Disposability”. Canadian Journal of Women and the Law, v. 28, pp- 285-307, 2016.

SCHEPER-HUGHES, Nancy y

BOURGOIS, Phillippe. Violence in War and Peace. An Anthology. Oxford: Blackwell Publishing, 2004.

SEGATO, Rita. La escritura en el cuerpo de las mujeres asesinadas en Ciudad Juárez. Territorio, soberanía y crímenes de segundo estado. Ciudad de México: Tinta y Limón, 2013.

SEODU HERR, Ranjoo. "Women's Rights as Human Rights and Cultural Imperialism". Feminist Formations, v. 31 n. 3, pp. 118-142, 2020.

SIEDER, Rachel y BARRERA, Ana. "Women and Legal Pluralism: Lessons from Indigenous Governance Systems in the Andes". Journal of Latin American Studies, v. 49, pp. 633-638, 2017.

SIERRA, María Teresa. "Derechos Humanos, Etnicidad y Género: Reformas Legales y Retos Antropológicos". En HERNÁNDEZ, Aida, PAZ, Sarela y SIERRA, María Teresa. El Estado y los indígenas en tiempos del PAN: Neoindigenismo, legalidad e identidad. CIESAS: México. pp. 307-332, 2004.

SUZACK, Cheryl, HUHNDROF, Sharin, PERREAULT, Jeanne y BARMAN, Jean. Indigenous Women and Feminism. Politics, Activism, Culture. Vancouver: UBCpress, 2010. 
TZUL TZUL, Gladys. “Sistemas de gobierno comunal indígena: la organización de la reproducción de la vida". El Apantle. Revista de Estudios Comunitarios, n. 1, pp. 125-140, 2015.

VIVEROS VIGOYA, Mara. "La interseccionalidad: Una aproximación situada a la dominación". Debate Feminista, n. 52, pp. 1-17, 2016.

WRIGHT, Melissa. Disposable women and other myths of global capitalism. New York: Routledge, 2006.

WRIGHT, Melissa. "Epistemological Ignorances and Fighting For the Dissapeared: Lesson from Mexico". Antipode, v. 49 n.1, pp. 5-25, 2017.

WRIGHT, Melissa. 'Necropolitics, Narcopolitics, and Femicide: Gendered Violence on the Mexico-U.S. Border". Signs: Journal of Women in Culture and Society, v. 36 n. 3, pp. 707-731, 2011.

\section{Sobre las autoras}

\section{Marisol Alcocer Perulero}

Es doctora en Ciencias Sociales, con mención en sociología, por la FLACSO, México, y realizó la Maestra en Estudio Culturales, por el Colegio de la Frontera Norte, Tijuana, B.C. Realizó una estancia de investigación posdoctoral en el Centro de Investigación y Estudios Superiores en Antropología Social. Está realizando la Investigación: "Vulnerabilidades y violencias diferenciadas contra mujeres de regiones con población mayoritariamente indígena y afromexicana en Guerrero" 20182019. Ha realizado estancias internacionales de investigación, la más reciente University Of California, Santa Cruz, con Ph.D RosaLinda Fregoso Research Professor/Professor Emeritus, para Discusión desde el enfoque de la interseccionalidad, mayo -junio de 2017. Ha sido parte de la primera investigación que se realizó a nivel nacional en torno al feminicidio en 10 entidades de la república, titulada "Violencia Feminicida en Guerrero", en 2006; además fue Integrante del grupo de trabajo que fue conformado para atender y posteriormente declarar la solicitud de Alerta de Violencia de Género en Guerrero.

\section{Katy Cedeño Peralta}

Joven indígena Miskitu e Ingeniera Agroforestal con formación en Ciencias Agroforestales del Trópico Húmedo de la Bluefields Indian and Caribbean University (BICU). Se ha desempeñado por más de 5 años en el Instituto de Investigación y Desarrollo "Nitlapan", adscrito a la Universidad Centroamericana (UCA) en el programa de investigación de la Costa Caribe norte; desde este espacio ha realizado estudios en las comunidades Indígenas de Kisalaya, Awastigni, Iltara, Betania en la línea de Investigación Medios y Estrategias de vida y su relación con la seguridad alimentaria. Así mismo, trabajó en procesos 
de desarrollo en la ejecución de la iniciativa de desarrollo Creando capacidades y oportunidades en Mujeres y jóvenes de la costa Caribe en Nicaragua en varios componentes entre ellos iniciativas económicas (GAPS). Además de participar en un proceso de aprendizaje denominado "Escuela Intercultural de Aprendizaje" en la que tiene como fin el rescate- revitalizar de la cultura e identidad Miskitu con Jóvenes de las comunidades indígenas en la que se realiza procesos de investigación acción.

\section{Natalia De Marinis}

Es profesora investigadora del Centro de Investigaciones y Estudios Superiores en Antropología Social (CIESAS), sede Golfo. Doctora en Antropología por el CIESAS, sede Cdmx. Es autora del libro Desplazadas por la guerra: Estado, género y violencia en la región triqui, publicado por CIESAS (2019) y coeditora, junto con Morna Macleod, del libro Resisting Violence: Emotional Communities in Latin America, publicado por Palgrave macmillan (2018). Ha publicado artículos sobre mujeres indígenas, interseccionalidad, violencia, seguridad, desplazamiento forzado y márgenes de Estado. Actualmente, realiza una investigación sobre violencia contra mujeres indígenas y acceso a la justicia en la región de Zongolica, Veracruz.

\section{Dolores Figueroa Romero}

Es catedrática CONACYT adscrita al Centro de Investigaciones y Estudios Superiores en Antropología Social (CIESAS) en la Ciudad de México. Sus principales líneas de investigación son las relaciones de género a lo interno de las organizaciones indígenas mixtas, procesos de participación política de mujeres indígenas en procesos electorales y la construcción subjetiva como defensoras de derechos humanos y mediadoras de discursos de derechos a diferentes niveles de gobernancia política. En los últimos dos años ha tomado parte en colaboración con la Dra. Sierra en el desarrollo del Laboratorio de Observación Etnográfica contra mujeres indígenas desde una perspectiva que enfatiza la intersección de varios órdenes de discriminación. Este Laboratorio tiene casa en el CIESAS. También es autora junto con la Dra. Arelly Barbeyto del ensayo "Strong Women: Memories of Miskitu Women forging Peace and Autonomy" publicado en el 2019 del libro: Indigenous Struggles for Autonomy. The Caribbean Coast of Nicaragua publicado por la editorial Lexington, UK.

\section{Selmira Flores}

Ciencias Sociales en la Universidad Nacional Autónoma de Nicaragua, una maestría en Estudios del Desarrollo del Colegio de Postgraduados de Montecillo del 
Estado de México y un Doctorado en Estudios del Desarrollo del Instituto de Políticas para el Desarrollo de la Universidad de Amberes de Bélgica. Ha trabajado en el sector de las Organizaciones No Gubernamentales, con proyectos para mujeres y comunidades rurales. Desde el 2002 trabaja como investigadora en el Instituto de Investigación y Desarrollo Nitlapan de la Universidad Centroamericana donde actualmente funge como directora de investigación. Ha trabajado investigación sobre el acceso de pequeños productores-as al mercado, cadenas de valor y género, gobernanza forestal y género en comunidades indígenas, el acceso de las mujeres y jóvenes a la tierra en el marco de la desigualdad y los conflictos por el manejo de los recursos.

\section{Perla O. Fragoso Lugo}

Doctora en Antropología por el Centro de Investigaciones y Estudios Superiores en Antropología Social (CIESAS, CDMX). Autoadscrita a un feminismo que se define principalmente por sus prácticas, por su ética y por su constante reflexividad. Actualmente es investigadora de Cátedras Conacyt comisionada al CIESAS-Peninsular, donde desarrolla una investigación sobre violencia de género contra las mujeres, violencia feminicida y feminicidio en Yucatán. De septiembre de 2014 a junio de 2018 participó en un proyecto colectivo sobre estas problemáticas en Chiapas, en el CESMECAUNICACH. Su libro A puro golpe. Violencias $y$ malestares sociales en la juventud cancunense (2016) fue merecedor del Premio INAH Fray Bernardino de Sahagún correspondiente al área de Etnología y Antropología Social en la categoría de Mejor Investigación. Miembro del Sistema Nacional de Investigadores Nivel I. Ha sido profesora en el posgrado en Estudios e Intervención Feministas del CESMECA-UNICAH, en el posgrado en Ciencias Sociales y Humanísticas de la misma institución, en la maestría en Antropología social en CIESAS-CDMX y en la maestría en Antropología en del IIA de la UNAM, entre otras instituciones. Ha participado como perita antropóloga en casos de feminicidio y violaciones a derechos humanos, fundamentalmente en contextos indígenas en Chiapas. Sus intereses de estudio han sido la antropología de las violencias, las violencias de género contra las mujeres, las juventudes, los estudios urbanos y la antropología de las emociones.

\section{Paulina García-Del Moral}

Doctora en Sociología por la Universidad de Toronto, Canadá. Su investigación de examina la intersección de la ley, el género y el poder en los procesos transnacionales de búsqueda de justicia en México y Canadá. García trabaja en tres áreas empíricas principales: el impacto del activismo 
feminista transnacional en las respuestas estatales al asesinato de mujeres; los viajes transnacionales y la institucionalización legal de los conocimientos feministas; y cómo las decisiones de las instituciones supranacionales de derechos humanos sobre las políticas de los estados sobre violencia de género y derechos reproductivos configuran las políticas nacionales de pertenencia.

\section{Arelly Mabell Barbeyto Rodríguez}

Realizó estudios de licenciatura en Sociología por la Universidad Centroamericana (UCA), estudios de maestría en Antropología Social graduada en la Universidad de las Regiones Autónomas de la Costa Caribe Nicaragüense (URACCAN) y estudios doctorales en Ciencias Sociales en el Programa Centroamericano de Postgrado en Ciencias Sociales de la Facultad Latinoamericana de Ciencias Sociales (FLACSO) sede académica Guatemala. Se desempeñó como docente e investigadora en URACCAN recinto Bilwi- Kamla, docente en la Bluefields and Indian and Univesity (BICU) núcleo Bilwi y miembro del comité editorial de NITLAPAN- UCA en Bilwi. Investiga en los campos de autonomía, género, violencia, pueblos indígenas y realidades socioculturales como sus áreas centrales de interés profesional y personal.

\section{Nora Esperanza Sánchez Castellón}

Originaria de San Juan de Limay, Departamento de Estelí, con domicilio en Costa caribe norte. de profesión Ingeniera Agropecuaria con Maestría en Pedagogía con mención en Docencia Universitaria. Se ha desempeñado por más de 4 años en el Instituto de Investigación y Desarrollo "Nitlapan", adscrito a la Universidad Centroamericana (UCA) en el programa de investigación de la Costa Caribe norte; en la actualidad es su coordinadora territorial. Desde este espacio ha conducido procesos sobre: Medios y Estrategias de vida y su relación con la seguridad alimentaria, desde el instituto en la sede caribeña se promueven iniciativas de desarrollo "Creando capacidades y oportunidades en Mujeres y jóvenes de la costa Caribe en Nicaragua". Además de impulsar la "Escuela Intercultural de Aprendizaje" enfatizando el reconocimiento y orgullo de la cultura e identidad Miskitu con Jóvenes indígenas en investigación aplicada.

\section{María Teresa Sierra Camacho}

Especialista en antropología jurídica y política, justicia de género y pluralismo jurídico. Fundadora de la Red Latinoamericana de Antropología Jurídica (RELAJU). Integrante de redes nacionales e internacionales en defensa de los derechos 
humanos de los pueblos indígenas, el antirracismo y en contra de las violencias de género. Se posiciona por una antropología colaborativa que vincula la academia con los procesos sociales. Profesora Investigadora Titular del CIESAS, adscrita a la institución desde 1984, y ha sido Investigadora visitante en el Center for US and Mexican Studies de la Universidad de California en San Diego (UCSD), octubre 2011-mayo 2012.

\section{Vivian Jiménez Estrada}

Es Maya Achí, nacida en Ixim Ulew (Guatemala). Es profesora-investigadora en el Departamento de Sociología de la Universidad de Algoma, Canadá, en donde reside actualmente en Baawaating, territorio Anishinaabe. Se dedica a la investigación, la enseñanza y el aprendizaje basados en conocimientos y perspectivas Indígenas. Ella trabaja localmente con el colectivo de mujeres Indígenas de Baawaating que lucha contra la Violencia hacia las Mujeres Indígenas (Sault Ste. Marie, Ontario).

\section{Norma Don Juan Pérez}

Pertenece al pueblo nahua, originaria de la Ciudad de México. Es integrante de la Coordinadora Nacional de Mujeres Indígenas (CONAMI México) desde el año 2009. Durante el período 2016-2019 fue parte del Consejo Colegiado del Enlace Continental de Mujeres Indígenas de las Américas (ECMIA). Actualmente, forma parte del Grupo Asesor de la Sociedad Civil (GASC) para el Foro Generación Igualdad México, que es el proceso de evaluación y generación de estrategias para el cumplimiento y avance de la Plataforma de Beijing. Es promotora de derechos humanos, educadora popular e investigadora social. Email: yoloyotl@gmail.com.

\section{Patricia Torres Sandoval}

Es integrante de la Organización Nación P'urhépecha Zapatista (ONPZ), ex integrante de la Coordinación general de la Coordinadora Nacional de Mujeres Indígenas de México (CONAMI), ahora integrante del Consejo de Mayoras de la misma organización. Cofundadora e integrante activa de la Red Nacional de Abogadas Indígenas (2011) y de la Red de Abogadas y Promotoras Indígenas por una Vida Libre de Violencia en el estado de Michoacán (2016). Email: dpattyt@hotmail.com 